\title{
Single Dose Bioequivalence Study of Two Rivaroxaban Tablet Formulations, Administered Orally after Being Crushed and Suspended in Apple Puree
}

\author{
Simona Nicoleta Duna ${ }^{1 *}$, Adrian Ghita ${ }^{1}$, Adelina Ciuciuleaca ${ }^{2}$, Irene Manzanera ${ }^{3}$, David Puibert ${ }^{3}$, Simona \\ Rizea-Savu ${ }^{4}$
}

13S-Pharmacological Consultation $\mathcal{E}$ Research SRL, Bucharest, Romania; ${ }^{2}$ Pharma Serv International SRL, Bucharest, Romania; ${ }^{3}$ Ferrer Internacional S.A. (Ferrer Grupo), Barcelona, Spain; ${ }^{3}$ S-Pharmacological Consultation $\mathbb{E}$ Research GmbH, Harpstedt, Germany

\begin{abstract}
The bioavailability of an active substance might be altered when a solid oral dosage form (SODF) is crushed or disintegrated and mixed with fluids or food in order to assist swallowing. In consequence, the current European Medicines Agency (EMA) practice is to request comparative bioavailability testing for bridging safety and efficacy data from a formulation administered whole to the same product administered crushed. Specific criteria for waiving in vivo testing of crushed products are available only for BCS Class I and Class III drugs. Since rivaroxaban is a Class II drug which can be administered crushed and mixed with fluids, any generic formulation has to be tested against the originator in this setting. Therefore, an open label, randomized, single dose, two-period, two-sequence, crossover bioequivalence study with administration of rivaroxaban $10 \mathrm{mg}$ crushed tablets suspended in apple puree was conducted in 24 fasting healthy volunteers. Both rivaroxaban treatments were well tolerated by the study subjects. A standardized, dose efficient and fully reproducible protocol for grinding, mixing and administering investigational medicinal products was developed and applied. Assessment of bioequivalence was based on plasma concentrations of parent rivaroxaban, quantified using a validated HPLC/MS/MS method. The $90 \%$ confidence intervals for $\mathrm{C}_{\max }$ and $\mathrm{AUC}_{0-\mathrm{t}}$ least square mean $\mathrm{T} / \mathrm{R}$ ratios were within the bioequivalence acceptance range of $80 \%$ to $125 \%$. Results from the present study reinforced the bioequivalence conclusion reached for the same test and reference products following administration of whole tablets in fasting state. No notable changes in bioavailability occurred when rivaroxaban tablets were crushed, immediately dispensed in $70 \mathrm{~mL}$ apple puree and quantitatively (entire dose recovered) administered to fasting volunteers.
\end{abstract}

Keywords: Rivaroxaban; Crushed tablets; Crushed versus whole; Comparative bioavailability; Generic; Bioequivalence

\section{INTRODUCTION}

Rivaroxaban is a highly selective direct factor Xa inhibitor which exerts its antithrombotic effect by interrupting the intrinsic and extrinsic pathway of the blood coagulation cascade, thus inhibiting thrombin formation and development of thrombi [1]. For patients who are unable to swallow whole tablets, the originator rivaroxaban (Xarelto of Bayer AG, Germany) provides the option of crushing the tablets and mixing them with water or apple puree immediately prior to their oral administration. Since the bioavailability of an active substance may be altered if a solid oral dosage form (SODF) is crushed or disintegrated and mixed with fluids or food in order to assist swallowing, the current European Medicines Agency (EMA) practice is to request comparative bioavailability testing for bridging safety and efficacy data from the formulation administered whole to the crushed product [2]. For innovative products, the bioavailability comparison must be carried between the SODF taken whole versus the same dosage form crushed and mixed with the fluid/food to be included in the SmPC recommendations for administration. For generics, bioequivalence to the originator must be shown for the SODF administered whole and also for at least one of the crushed-mix alternatives available in the SmPC of the originator. This requirement stems from the observation that a test product that is shown to be bioequivalent when administered as a whole tablet in fasted state may exhibit significantly different bioavailability compared to the reference product when both are administered crushed/disintegrated and dispersed in food [2]. The likelihood of a formulation-specific change in bioavailability at

Correspondence to: Simona Nicoleta Duna, Research Project Manager, 3S-Pharmacological Consultation \& Research SRL, Romania, Tel: +40 758109 202; E-mail: nicoleta.duna@3spharma.eu

Received: January 29, 2019, Accepted: February 18, 2019, Published: February 25, 2019

Citation: Duna SN, Ghita A, Ciuciuleaca A, Manzanera I, Puibert D, Savu SR (2019) Single Dose Bioequivalence Study of Two Rivaroxaban Tablet Formulations, Administered Orally after Being Crushed and Suspended in Apple Puree. J Bioequiv Availab 11:388. doi: 10.35248/0975 0851.19.11.388

Copyright: (C) 2019 Duna SN, et al. This is an open-access article distributed under the terms of the Creative Commons Attribution License, which permits unrestricted use, distribution, and reproduction in any medium, provided the original author and source are credited. 
crushing is considered low enough to waive bioequivalence testing in crushed state only if the following conditions are simultaneously met: bioequivalence of uncrushed products is demonstrated, the active ingredient is highly soluble and stable in the $\mathrm{pH}$-range of the gastrointestinal tract according to BCS-based biowaiver criteria (drugs included in Class I or III); surface active excipients do not differ qualitatively nor quantitatively between test and reference and no specific formulation characteristics or manufacturing technologies differ; disintegration time is comparable between test and reference; dissolution profiles are similar between test and reference product at $\mathrm{pH} \mathrm{1.2,4.5} \mathrm{and} \mathrm{6.8.} \mathrm{Being} \mathrm{a} \mathrm{BCS} \mathrm{Class} \mathrm{II}$ drug [3], rivaroxaban did not fulfil the waiver criteria for crushed bioequivalence testing. Therefore, after successful demonstration of bioequivalence between the test product (Rivaroxaban 10 mg film-coated tablets of Ferrer Internacional S.A., Spain) and Xarelto $^{\circledR} 10 \mathrm{mg}$ film coated tablets in a single-dose pivotal study with administration of whole tablets in fasting state, a second study with crushed tablets was also performed. The latter was an open label, two-period, two-sequence, two-way crossover, randomized, single dose bioequivalence study with crushed rivaroxaban tablets, conducted in healthy volunteers under fasting conditions. One of the biggest challenges faced during pre-trial preparations was the development of a standardized procedure for crushing the tablets, mixing the disintegrated particles with apple puree and administering the resulting suspension without product loss, crosscontamination or risk of bias due to an uneven size of crushed particles. The first step of this process involved the testing of 15 different tablet crushing or grinding devices commonly used in clinical practice (ranging from hand-held lever or rotating crushers made of plastic or metal, mortar and pestle, bench top manually operated vertical or horizontal pressing devices, and electric pill grinders). The criteria for evaluation of suitability were: likelihood of variability stemming from multiple repetitions of the procedure (devices that require application of force by a human operator yield good results in terms of particle size for the first subjects, but performance is likely to decrease over time due to tiredness), recoverability of the grinded product, availability of single use accessories, and homogeneity of crushed particles in terms of size. The best results were obtained with one of the automated professional pill grinders, which was subsequently used in the study according to very detailed instructions for standardization of dose preparation and administration (see Materials and Methods).

\section{MATERIALS AND METHODS}

\section{Study design and participants}

An open label, randomized, single dose, two-period, two-sequence, crossover bioequivalence study with administration of crushed tablets suspended in apple puree was conducted in 24 fasting healthy male and female volunteers. All subjects were adults (18 years or older) with a body mass index within 18.5 to $30.0 \mathrm{~kg} / \mathrm{m}^{2}$, who did not suffer from rare hereditary problems of glucose intolerance, the Lapp lactase deficiency, glucose-galactose malabsorption or gluten intolerance. All subjects gave their written informed consent before they underwent any study-related procedures and were free to withdraw from the trial at any time. The study medication administration consisted of one single $10 \mathrm{mg}$ rivaroxaban filmcoated tablet (test or reference formulation) in each study period, with a washout period of 10 days between crossover doses. Both study products were orally administered crushed and mixed with $70 \mathrm{~mL}$ apple puree, after at least 10 hours of overnight fasting (see Investigational Products and Administration Method). Except for $130 \mathrm{~mL}$ of rinsing still bottled water administered after intake of the rivaroxaban-puree suspension, drinking of water or other fluids was restricted from one hour prior to dosing until one hour post dosing. For the analytical determination of rivaroxaban plasma levels, venous blood samples of $5 \mathrm{~mL}$ were drawn in tubes containing $\mathrm{K}_{3}$ EDTA as anticoagulant before study drug administration and at $0.167,0.50,0.75,1.00,1.50,2.00,2.50$, $3.00,3.50,4.00,4.50,5.00,5.50,6.00,7.00,8.00,10.00,12.00$, $16.00,24.00,36.00,48.00$ and 72.00 hours post dose in each study period. The primary pharmacokinetic parameters considered for bioequivalence assessment were $\mathrm{AUC}_{0 . t}$ and $\mathrm{C}_{\max }$.

The study was conducted following unconditional approval from the National Ethics Committee, the Ethics Committee of the Clinical Hospital of the Ministry of Health of the Republic of Moldova and the Medicine Agency. Clinical investigations were conducted according to the Declaration of Helsinki principles and Good Clinical Practice.

\section{Investigational products and administration methods}

The Reference product, Xarelto ${ }^{\circledR} 10 \mathrm{mg}$ film coated tablets (Bayer Schering Pharma AG, Germany) was sourced from the German market. The Test product, Rivaroxaban $10 \mathrm{mg}$ film-coated tablets, was manufactured by Ferrer Internacional S.A. (Ferrer Grupo) in a GMP production facility located in Spain.

In each study period either one crushed film-coated tablet of test or one crushed film-coated tablet of reference, suspended in $70 \mathrm{~mL}$ apple puree, was administered to each subject. Dose preparation and administration: Preparation of the apple puree - crushed tablet suspension was done for each subject within 5 minutes prior to dosing. A new $100 \mathrm{~mL}$ apple puree pouch was unsealed for each subject and a volume of $70 \mathrm{~mL}$ puree at room temperature was precisely measured in a $100 \mathrm{~mL}$ tall Berzelius beaker. A new glass beaker was used for each subject. After a thorough check of individual labeling, identity and integrity of each tablet, it was placed in a single use grinding cup and covered with a single use round foil. Then the whole ensemble was inserted in the professional pill grinder and submitted to a full automated grinding cycle. The grinded rivaroxaban was transferred to the tall Berzelius beaker containing $70 \mathrm{~mL}$ apple puree and mixed vigorously for precisely 30 seconds. The labeled administration beaker, the utensil used for mixing, the individual packaging of the tablet used for preparation, the cup and the discus used for grinding were all placed in a single use tray and handed over to the administration team. The team supervising the administration was comprised of two physicians (the Clinical Investigator and Study Monitor). Before handing the glass beaker to the subject for self-administration, the beaker and secondary package labeling were checked once more against the randomization table and subject bracelet and tag. Once the subjects were handed the rivaroxaban in apple puree suspension and told that they could start to eat, full intake was achieved within 1 minute. After full intake of the rivaroxaban-apple puree suspension, the administration beaker and teaspoon as well as the grinding cup and disk were rinsed with $65 \mathrm{~mL}$ of water which was drank by the subject; then, the procedure was repeated once more 
bringing the total volume of room-temperature rinsing still bottle water drank by each subject to $130 \mathrm{~mL}$. All the transparent single use labware used for preparation and dosing was clean after the second rinsing cycle, thus confirming that the full medication dose was successfully administered to each subject (Figure 1).

\section{Handling and bioanalysis of study samples}

For the quantification of rivaroxaban, venous blood samples of approximately $5 \mathrm{~mL}$ were collected in labelled tubes containing $\mathrm{K}_{3}$ EDTA as anticoagulant. After collection, the blood samples were centrifuged under refrigeration (10 minutes at $1500( \pm 5) \mathrm{g}$ and a nominal temperature of $4^{\circ} \mathrm{C}$ ). Plasma was separated, divided into duplicate aliquots and, within 60 minutes from collection, frozen for storage at $-20^{\circ} \mathrm{C}$ nominal until shipped to the analytical laboratory. Plasma samples (first aliquot) were sent from the clinical site to the analytical facility in a thermo-insulated box containing an adequate amount of dry ice. During transport, an electronic logger was used for monitoring plasma samples temperature. Once received at the analytical laboratory, the samples were stored at $-20^{\circ} \mathrm{C}$ or colder until submitted to analysis. Before analysis, plasma samples were thawed, mixed for 3 minutes and centrifuged for 3 minutes at $4000 \mathrm{rpm}$ and $20^{\circ} \mathrm{C}$ nominal. Aliquots of samples were spiked with internal standard $\left(\left[{ }^{13} \mathrm{C}_{6}\right]\right.$-rivaroxaban $)$, diluted, vortexed and centrifuged. Supernatants were diluted with ammonium acetate in water $+0.1 \%$ formic acid solution, mixed and centrifuged again. Finally, the samples have been transferred to the autosampler, awaiting injection. The analytical and internal standard used for HPLC-MS/MS determinations were rivaroxaban and respectively $\left[{ }^{13} \mathrm{C}_{6}\right]$-rivaroxaban (both purchased from AlsaChim, France). Commercially available reagents of analytical/HPLC grade purity were used for sample processing. Eight-point calibration curves were prepared in appropriate matrix (pooled human plasma deriving from blank blood samples collected on $\mathrm{K}_{3}$ EDTA from healthy volunteers were spiked with internal standard, processed and diluted according to the same protocol previously described for study samples). The concentration range of the calibration curve was $1.0-800.0 \mathrm{ng} / \mathrm{mL}$ (lower limit and respectively upper limit of quantification for the analytical method). Spiked QC samples were prepared at the following concentrations: 3.0, 64.0, 160.0, 320.0 and $640.0 \mathrm{ng} / \mathrm{mL}$. Calibration curves and QC samples were analyzed during each analytical sequence. Analyses were carried out on a MPX-2 multiplexing HPLC system (Applied BiosystemsSciex, Canada) comprised of an autosampler (HTS PAL of CTC Analytics, Switzerland) with 2 injection valves that permitted injection into 2 separate HPLC columns, each of them connected to a high pressure gradient system (Schimadzu Prominence of Shimadzu, Japan). A selector valve was connected to the exit of each column, permitting to alternatively introduce the mobile phase in the LC-MS source. The software MPX-2 controlled all functions of the multiplexing, keeping a clear audit-trail of the operations, sample by sample. A triple quadrupole MS/MS mass spectrometer model API 6500 (Applied Biosystems-Sciex, Canada) equipped with atmospheric pressure electrospray ionization interface (TurboSpray ion Drive) was used. Each analytical sequence, composed of calibration curve, quality controls and study samples, was injected in a single column, to avoid the risk of differences in quantitation between the 2 columns, and 2 sequences were run in parallel. To minimize idle time between injections only the periods of peaks elution of each sample were acquired, and samples were injected overlapping the initial part of the chromatograms as well as the
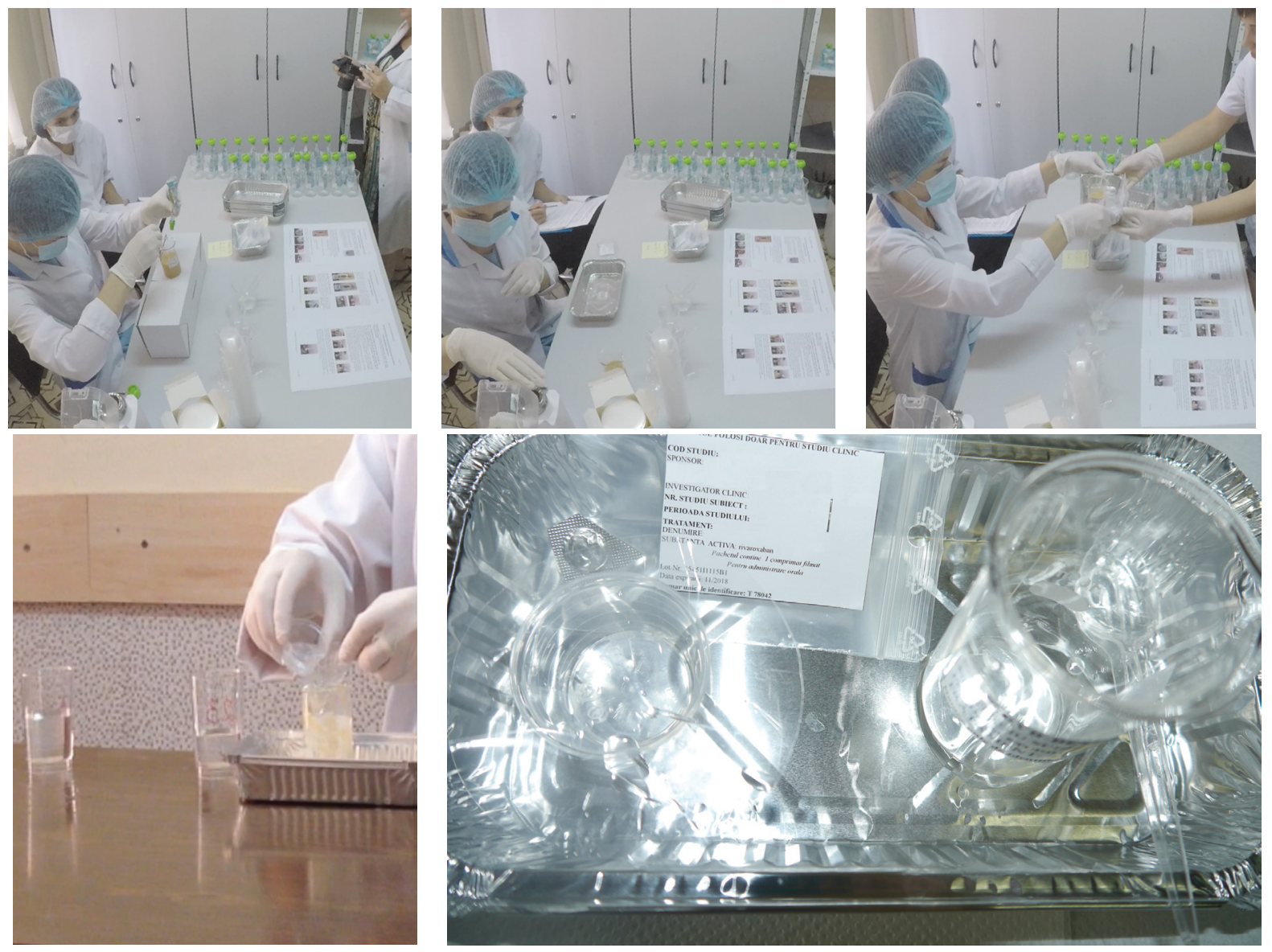

Figure 1: Representative snapshots of the preparation and rinsing processes 
final equilibration, when data acquisition was not needed, with the recording of peaks from the sample running in parallel.

Reversed phase analytical columns Ascentis Express C18, 10 cm* $2.1 \mathrm{~mm}, 2.7 \mu \mathrm{m}$ silica packing (Supelco, Germany) were used, heated at $40^{\circ} \mathrm{C}$ nominal. The sample extracts were injected on the column eluted at $0.4 \mathrm{~mL} / \mathrm{min}$. Separations were carried out in isocratic conditions. The injection volume was $50 \mu \mathrm{L}$. Quantitative data were acquired in positive ions mode using a multiple reaction monitoring method (MRM). The MRM transitions considered were 436.032/ 145.000 for rivaroxaban and 442.021/ 145.000 for $\left[{ }^{13} \mathrm{C}_{6}\right]$-rivaroxaban. The analytical method was fully validated as per the latest EMA and FDA requirements [4,5] before the start of analysis of the study plasma samples. The method was verified for linearity, quantification limits, assay specificity, between-run and within-run precision and accuracy, analyte recovery, and stability in stock solution and biological matrix under processing conditions during the entire period of storage. The analytical protocol was prepared prior to the start of bioanalysis. Analytical work was performed blindly (without access to the randomization list) and according to Good Laboratory Practices (GLP). The bioassay reproducibility was assessed through incurred samples reanalysis.

\section{Pharmacokinetic and statistical analysis}

Non-compartmental PK analysis was performed using $\mathrm{SAS}^{\circledR}$ statistical software, version 9.4 (SAS Institute Inc., USA).Maximum plasma concentration $\left(\mathrm{C}_{\max }\right)$ and time to reach maximum plasma concentration $\left(\mathrm{T}_{\max }\right)$ were obtained directly from the plasma values (observed, not calculated). The linear trapezoidal rule was used to calculate the area under the concentration-time curve from time zero to the last quantifiable concentration $\left(\mathrm{AUC}_{0-\mathrm{t}}\right)$. The apparent elimination rate constant $\left(\mathrm{K}_{\mathrm{el}}\right)$ was estimated by regression of the terminal ln-linear portion of the plasma concentration-time profile; apparent terminal half-life $\left(\mathrm{t}_{1 / 2}\right)$ was calculated as the quotient of $\ln (2)$ and $\mathrm{K}_{\mathrm{el}}$. Area under the curve to infinity $\left(\mathrm{AUC}_{0_{-\infty}}\right)$ was estimated as the sum of $\mathrm{AUC}_{0-\mathrm{t}}$ and the extrapolated area given by the quotient of the last quantifiable plasma concentration and $\mathrm{K}_{\mathrm{el}}$. ANOVA was performed on In-transformed $\mathrm{C}_{\max }, \mathrm{AUC}_{0 \mathrm{t}}$ and $\mathrm{AUC}_{0-\infty}$ using the General Linear Models (GLM) procedure fitted in $\mathrm{SAS}^{\circledR}$ software (version 9.4) using the method of least squares. The confidence interval for the ratio of the population means was calculated considering a classic (shortest) 90\% confidence interval. The bioequivalence acceptance range was set to $80.00-125.00 \%$ for rivaroxaban. The effect of sequence, period, subject within sequence and treatment on rivaroxaban $\mathrm{C}_{\max }, \mathrm{AUC}_{0-\mathrm{t}}$ and $\mathrm{AUC}_{0-\infty}$ has been separately evaluated on ln-transformed data. The intrasubject variability (ISCV) of $\mathrm{C}_{\max }, \mathrm{AUC}_{0-\mathrm{t}}$ and $\mathrm{AUC}_{0-\infty}$ was also separately determined. The bioequivalence comparison has been carried out on the primary pharmacokinetic parameters $\mathrm{C}_{\max }$ and $\mathrm{AUC}_{0-\mathrm{t}}$, while the data presented for $\mathrm{AUC}_{0_{-\infty}}$ can be regarded as supporting evidence. $\mathrm{T}_{\max }$ data have been compared using the non-parametric Wilcoxon Signed-Rank Test. The test was applied to untransformed data. The limit of statistical significance was considered $\mathrm{p}<0.05$.

\section{RESULTS AND DISCUSSION}

\section{Pharmacokinetic results}

This study aimed to compare the relative bioavailability of one generic rivaroxaban film-coated tablet formulation versus the marketed reference product Xarelto ${ }^{\circledR}$, administered after being crushed and mixed with $70 \mathrm{~mL}$ apple puree. Since the SmPC of the reference product did not offer any specific information regarding the volume of fluid in which one crushed tablet should be dispersed, the administration conditions were selected in accordance to publicly available data from the bioequivalence study of Xarelto ${ }^{\circledR}$-crushed versus Xarelto ${ }^{\circledR}$-whole which was conducted by the originator company (crushed products suspended in a precisely measured $70 \mathrm{~mL}$ volume of apple puree) [6]. The preparation, administration and rinsing protocols developed in-house ensured the homogeneity of crushed particles and reduced to a minimum the risks of cross-contamination and product loss.

A total of 24 healthy male and female volunteers were enrolled in the study. All subjects were Caucasian with the mean age of 30.38 years (range $18-49$ years) and mean BMI of $24.10 \mathrm{~kg} / \mathrm{m}^{2}$ (range 18.7- $29.8 \mathrm{~kg} / \mathrm{m}^{2}$ ). After the first study period, two subjects exercised their right to withdraw from the study (for personal reasons, unrelated to the study medication). The Per Protocol Safety Population was comprised of all 24 subjects enrolled and treated with at least one dose of study medication (drop-outs after Period I included). The Per Protocol PK Population was comprised of the 22 subjects who completed both study periods and provided fully evaluable pharmacokinetic profiles. The mean rivaroxaban concentration-time curves after test and reference are shown in Figure 2 while mean pharmacokinetic parameters are summarized in Table 1.

The mean rivaroxaban pharmacokinetic curves were remarkably similar, to the point where scaling of the plots was needed (data display truncated at 48 hours) in order to develop graphs in which the reader can distinguish between the two almost superimposable profiles. The analysis of variance (ANOVA) did not show any statistically significant difference between the test and the reference formulations with respect to the fixed effects of period, sequence and treatment on the pharmacokinetic parameters analyzed $\left(\mathrm{C}_{\max }\right.$, $\mathrm{AUC}_{0-\mathrm{t}}$ and $\left.\mathrm{AUC}_{0-\infty}\right)$. The subject within sequence fixed effect was statistically significant $(p<0.05)$ for all three pharmacokinetic parameters analyzed. It is worth noting that this effect is almost always statistically significant as it only indicates that the enrolled subjects have different physiological characteristics [7], heterogeneity being key when a test group is intended to be representative for the general population. Identical median $\mathrm{T}_{\max }$ values were obtained for the two treatments (Table 1) and the statistical test (Wilcoxon Signed-Rank) applied to untransformed individual data further demonstrated that there is no statistically significant difference between treatments with respect to time needed to reach maximum plasmatic concentration. The point estimates of rivaroxaban pharmacokinetic. In-transformed parameters and the $90 \%$ confidence intervals for the ratios of the population means, along with the intra-subject CVs registered are shown in Table 2.

The statistical evaluation of pharmacokinetic data presented herein shows that the two rivaroxaban formulations are bioequivalent as the test-reference ratios for the geometric means (\%) of the primary 

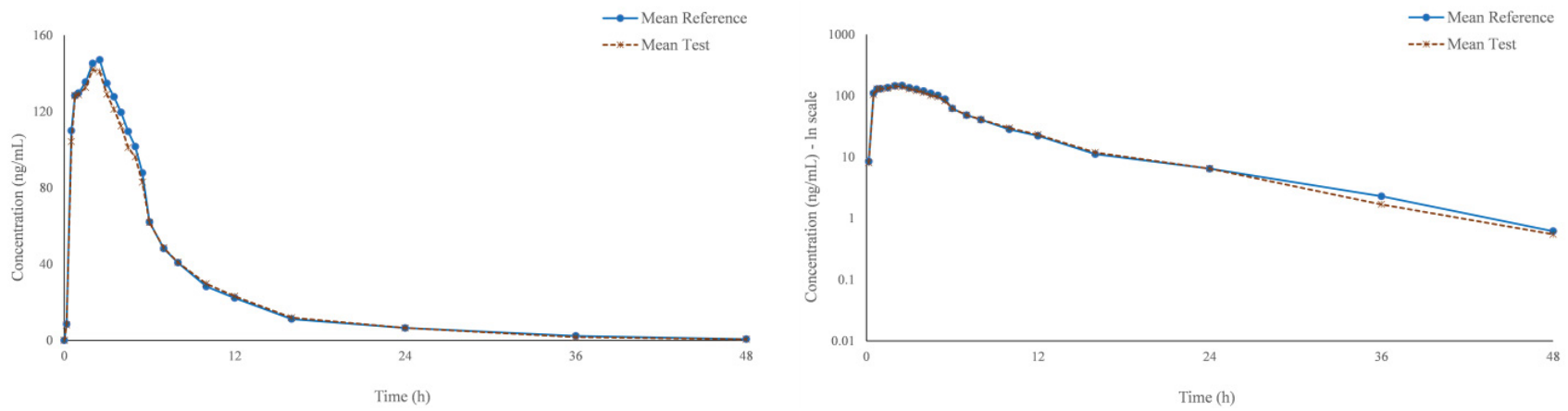

Figure 2: Rivaroxaban mean pharmacokinetic curves after test and reference (linear-linear and ln-linear display)

Table 1: Mean Pharmacokinetic Parameters for rivaroxaban $10 \mathrm{mg}$ crushed and mixed with $70 \mathrm{~mL}$ apple puree $(\mathrm{N}=22)$.

\begin{tabular}{cccr}
\hline PK parameter & Data presented & Test $(\mathrm{T})$ & Reference $(\mathrm{R})$ \\
\hline $\mathrm{C}_{\text {max }}(\mathrm{ng} / \mathrm{mL})$ & Mean $( \pm \mathrm{SD})$ & $158.357(29.752)$ & $160.454(42.644)$ \\
\hline $\mathrm{AUC}_{0+\mathrm{t}}\left(\mathrm{ng}{ }^{*} \mathrm{~h} / \mathrm{mL}\right)$ & Mean $( \pm \mathrm{SD})$ & $1077.053(280.839)$ & $1102.087(315.683)$ \\
\hline $\mathrm{AUC}_{0-\infty}\left(\mathrm{ng}^{*} \mathrm{~h} / \mathrm{mL}\right)$ & Mean $( \pm \mathrm{SD})$ & $1100.902(279.597)$ & $1124.025(317.889)$ \\
\hline $\mathrm{T}_{\text {max }}(\mathrm{h})$ & Median $($ Range $)$ & $2.0(0.5-3.0)$ & $2.0(0.5-5.0)$ \\
\hline $\mathrm{K}_{\mathrm{el}}(1 / \mathrm{h})$ & Mean $( \pm \mathrm{SD})$ & $0.103(0.037)$ & $0.106(0.052)$ \\
\hline$t_{1 / 2}(\mathrm{~h})$ & Mean $( \pm \mathrm{SD})$ & $7.904(4.014)$ & $7.927(3.262)$ \\
\hline
\end{tabular}

Table 2: Rivaroxaban point estimates, $90 \%$ CIs and ISCV for $\mathrm{C}_{\max }, \mathrm{AUC}_{\mathrm{ot}}, \mathrm{AUC}_{0-\infty}(\mathrm{N}=22)$.

\begin{tabular}{cccc}
\hline PK parameter & $\mathrm{T} / \mathrm{R}$ Ratio (\%) & 90\% Confidence Interval & Intra-subject CV (\%) \\
\hline $\mathrm{C}_{\max }$ & 100.05 & $92.24-108.53$ & 15.73 \\
\hline $\mathrm{AUC}_{0-\mathrm{t}}$ & 98.16 & $89.77-107.34$ & 17.32 \\
$\mathrm{AUC}_{0-\infty}$ & 98.47 & $90.28-107.40$ & 16.81 \\
\hline
\end{tabular}

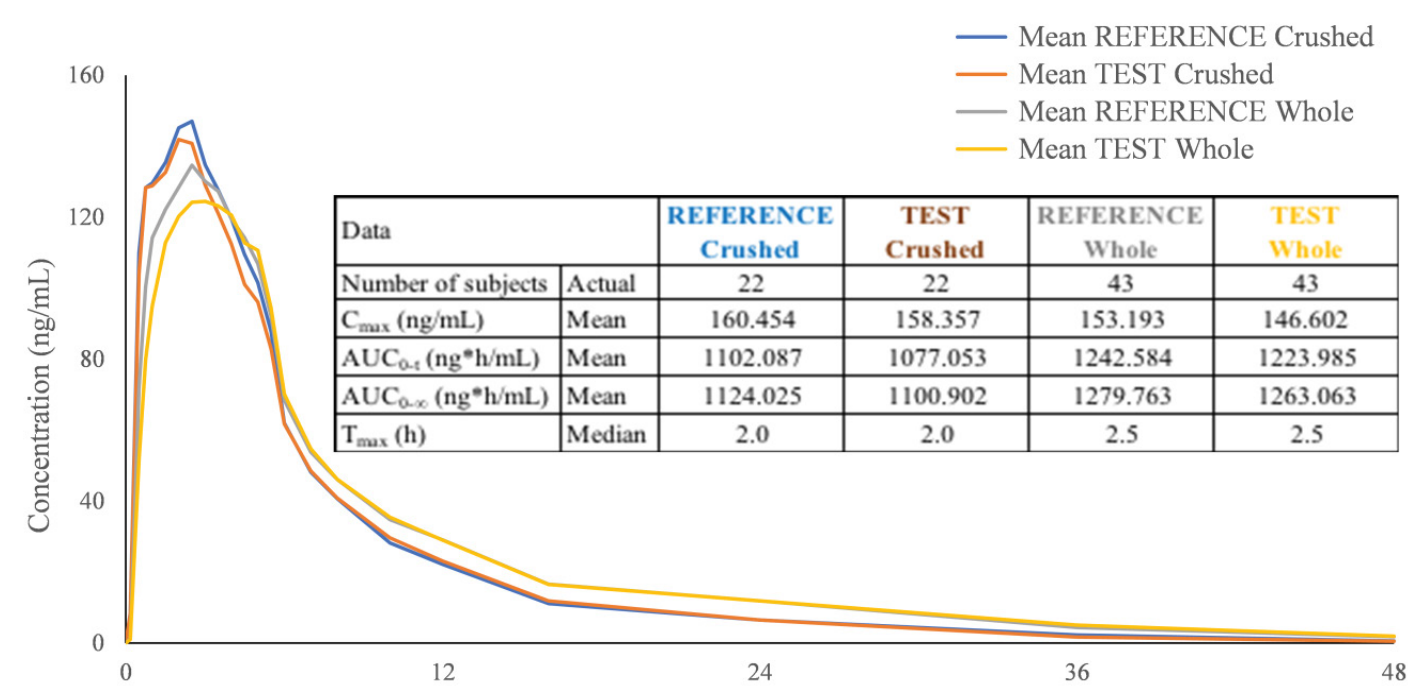

Time (h)

Figure 3: Rivaroxaban mean pharmacokinetic curves after test and reference administered crushed or whole, including a summary data table on PK parameters. Note: While individual data markers were removed for a better view of the four overlaid curves, it is important to note that the sampling time points were identical among studies.

parameters $\left(\mathrm{C}_{\max }\right.$ and $\left.\mathrm{AUC}_{0 . \mathrm{t}}\right)$ and their corresponding two-sided 90\% CIs were contained within the predefined regulatory limits of $80.00 \%$ to $125.00 \%$ [8]. Results from the statistical analysis of the additional pharmacokinetic parameter $\mathrm{AUC}_{0-\infty}$ further sustain the conclusion of equivalence between the two products. All individual extrapolated areas were well below the commonly accepted threshold of $20 \%$ (maximum observed being slightly below $6 \%$ for both formulations), thus demonstrating that the sampling schedule allowed for a proper evaluation of terminal elimination half-life and total extent of absorption. None of the subjects had measurable (above the $1.0 \mathrm{ng} / \mathrm{mL}$ LLOQ of the analytical method) levels of rivaroxaban at the second period pre-dose time point, confirming that the washout of 10 days was adequate for total elimination of the drug between subsequent administrations.

Results from this study are consistent with those obtained for the same formulations in a single dose study in which the tablets were administered whole, in fasting state (Figure 3). No notable changes in bioavailability occurred when rivaroxaban tablets were crushed, 
immediately dispensed in $70 \mathrm{~mL}$ apple puree and quantitatively (entire dose recovered) administered to fasting volunteers. Results from the present study reinforced the bioequivalence conclusion reached following administration of the test and reference whole tablets.

Peak rivaroxaban plasma levels were reached 30 minutes earlier and a mild increase was seen in mean $\mathrm{C}_{\max }(5 \%$ for reference, $8 \%$ for test) when comparing between administration of crushed versus whole tablets. When administered in crushed state, the higher rate of exposure was most likely owed to the fact that disintegration was already achieved by the time the micronized rivaroxaban formulations [3] reached the absorption site (the primary absorption site is the stomach, absorption being gradually reduced should drug release occur into the proximal small intestine or more distally into the small intestine or colon [9]). At the same time, total exposure $\left(\mathrm{AUC}_{0 . t}\right)$ was approximately $12 \%$ lower when comparing between administration of crushed versus whole tablets, due to the slower elimination observed in the whole tablets study (most likely because starting with the $10 \mathrm{mg}$ dose the elimination of rivaroxaban becomes absorption rate limited [3], mean terminal half-life being 3 hours longer in the whole tablets study where absorption was slower).

\section{Safety results}

Three adverse events occurred in one of the twenty-four subjects dosed at least once in the present study (Per Protocol Safety Population). Adverse events experienced by this subject (headache, nausea and vomiting) were mild in intensity and transient, complete recovery being concluded for all events within a maximum three hours from onset. Regardless of the formulation administered, a single dose of $10 \mathrm{mg}$ rivaroxaban was safe and well tolerated.

\section{CONCLUSION}

A standardized, dose efficient and fully reproducible protocol for grinding, mixing and administering investigational medicinal products was developed for application in bioequivalence studies in which it is required to dispense crushed SODFs suspended in fluids. The methodology was applied in a single dose bioequivalence study in mixed gender healthy volunteers with administration of $10 \mathrm{mg}$ rivaroxaban tablets crushed and suspended in apple puree. Though rivaroxaban itself is a BCS Class II drug exhibiting low solubility and high permeability, micronisation was used in the manufacturing process of the two study formulations in order to increase the particle surface area and facilitate dissolution. The statistical evaluation of pharmacokinetic data showed that the two rivaroxaban formulations (Xarelto ${ }^{\circledR} 10 \mathrm{mg}$ film coated tablets as reference and Rivaroxaban $10 \mathrm{mg}$ film-coated tablets of Ferrer Internacional S.A. as test) are bioequivalent as the testreference ratios for the geometric means (\%) of $\mathrm{C}_{\max }$ and $\mathrm{AUC}_{0 . \mathrm{t}}$ and their corresponding two-sided $90 \%$ CIs were contained within the predefined regulatory limits of $80 \%$ to $125 \%$. Presented in conjunction with data from a previous study in which the same test and reference were administered as whole tablets in fasting state, these results suggest that no notable changes in bioavailability occurr when rivaroxaban tablets are crushed, immediately dispensed in $70 \mathrm{~mL}$ apple puree and quantitatively (entire dose recovered) administered to fasting volunteers. Both rivaroxaban treatments, administered in single dose to healthy volunteers, were well tolerated.

\section{ACKNOWLEDGMENT}

The study was sponsored by Ferrer Internacional S.A. (Ferrer Grupo), Barcelona, Spain as manufacturer of the test product. The clinical, analytical, pharmacokinetic and statistical parts of the study were carried out by 3S Pharmacological Consultation $\&$ Research GmbH, Harpstedt, Germany through its affiliates (3S-Pharmacological Consultation \& Res. SRL and Pharma Serv International SRL, both located in Bucharest, Romania).

\section{REFERENCES}

1. SmPC of Xarelto $10 \mathrm{mg}$ film-coated tablets, Revised August 2018.

2. European Medicines Agency, CHMP, Clinical pharmacology and pharmacokinetics: questions and answers.

3. European Medicines Agency, CHMP (2008) Assessment Report for Xarelto, EMEA/543519/2008.

4. European Medicines Agency, CHMP (2011) Guideline on bioanalytical method validation, EMEA/CHMP/EWP/192217/2009 Rev. 1 Corr. $2^{* *}$.

5. Food and Drug Administration, Bioanalytical Method Validation Guidance for Industry, May 2018.

6. Moore KT, Krook MA, Vaidyanathan S, Sarich TC, Damaraju CV, Fields LE. Rivaroxaban Crushed Tablet Suspension Characteristics and Relative Bioavailability in Healthy Adults When Administered Orally or Via Nasogastric Tube. Clin Pharm in Drug Dev. 2014;3(4):321-327.

7. Luca Loprete. Common statistical remarks on bioequivalence regulatory submissions. Italian Society of Pharmaceutical Medicine Conference Proceedings, October 2013, Milan, Italy.

8. European Medicines Agency, Guideline on the investigation of bioequivalence, 2010, (EMA/CPMP/EWP/QPW/1401/98 Rev. 1 Corr. ${ }^{*}$.

9. Mani H, Kasper A, Last EL. Measuring the anticoagulant effects of target specific oral anticoagulants-reasons, methods and current limitations. J Thromb Thrombolysis. 2013;36(2):187-194. 